\title{
Inter-ELM evolution of the edge current density in JET-ILW type I ELMy H-mode plasmas
}

\author{
L. Horvath ${ }^{1}$, C.F. Maggi ${ }^{2}$, F.J. Casson ${ }^{2}$, V. Parail ${ }^{2}$, \\ L. Frassinetti ${ }^{3}$, F. Koechl ${ }^{2}$, S. Saarelma ${ }^{2}$, M.G. Dunne ${ }^{4}$, \\ K.J. Gibson ${ }^{1}$ and JET Contributors* \\ EUROfusion Consortium, JET, Culham Science Centre, Abingdon OX14 3DB, UK \\ ${ }^{1}$ York Plasma Institute, Department of Physics, University of York, York, \\ YO10 5DD, UK \\ ${ }^{2}$ CCFE, Culham Science Centre, Abingdon, OX14 3DB, UK \\ ${ }^{3}$ Division of Fusion Plasma Physics, KTH Royal Institute of Technology, \\ Stockholm, SE \\ ${ }^{4}$ Max-Planck-Institut für Plasmaphysik, Boltzmannstrasse 2, D-85748, Garching, \\ Germany \\ *See the author list of X. Litaudon et al 2017 Nucl. Fusion 57102001 \\ E-mail: laszlo.horvath@ukaea.uk
}

\begin{abstract}
.
Recent studies $[1,2]$ have shown that on JET with the Be/W ITER-like wall (JETILW) in high $\beta$ discharges with high $\mathrm{D}_{2}$ gas rates the inter-ELM temperature pedestal growth is saturated half way through the ELM cycle, leading to plasmas with reduced confinement, and that the linear MHD stability of these pedestals is inconsistent with the Peeling-Ballooning paradigm $[3,4]$. In this paper, the inter-ELM evolution of the edge current density is investigated in a wide range of type I ELMy H-modes on JETILW. It is found that in discharges at low gas rate the peak edge bootstrap current continuously increases until the ELM crash, while it saturates during the ELM cycle at high gas rates. The effect of current diffusion on the build-up of the edge current inter-ELM is assessed by simulating the Ohmic current contribution with the JETTO transport code. The simulations indicate that current diffusion contributes little to the time evolution of the total edge current in the second half of the ELM cycle and the total current is dominated by the bootstrap current. Therefore, current diffusion does not explain why JET-ILW type I ELMy pedestals at high gas rate and high $\beta_{N}$ are found to be stable to Peeling-Ballooning modes.
\end{abstract}

Keywords: H-mode, pedestal, ELMs, edge current, bootstrap current, current diffusion, JET-ILW 


\section{Introduction}

In a high confinement mode (H-mode) [5] tokamak plasma the energy and particle transport at the edge is reduced and a narrow region with steep pressure gradient is formed, called the pedestal. Optimising pedestal confinement is beneficial for fusion performance as high pedestal pressure leads to higher core pressure [6]. However, the steep pressure gradient in the pedestal can trigger Edge Localised Modes (ELMs) which are periodic instabilities of the plasma edge [7], followed by a transient loss of energy and particles. Consequently, ELMs lead to high heat loads on the plasma facing components and the resulting damage is a major concern in large scale devices. Thus, understanding the physical processes governing the behaviour of the pedestal is crucial in order to predict and optimise the plasma performance in future devices such as ITER [8].

A well-known theory which appears to explain the stability conditions in the pedestal is the peeling-ballooning (P-B) model [3, 4]. According to this model, the edge pressure gradient and current density drive coupled $\mathrm{P}-\mathrm{B}$ modes unstable, which trigger a type I ELM. The main drive for the ballooning modes is the pressure gradient, while the current density can stabilise ballooning modes through reduced magnetic shear. The current density can also drive peeling modes unstable and in the pedestal it is typically dominated by the bootstrap current [9], which can be efficiently driven by the steep pressure gradient. The EPED model $[10,11]$ is capable to give predictions for both the height and the width of the pedestal by combining the stability of P-B modes and Kinetic Ballooning Modes (KBMs). It assumes that the edge pressure gradient is locally constrained by KBMs in the inter-ELM cycle, and the pedestal width can further evolve (with a limited gradient), until the P-B mode is triggered. This, in most cases, leads to the crash and the pedestal collapses.

A recent study has shown that the pedestal stability in JET with the Be/W ITERlike wall (JET-ILW) is consistent with the P-B model in discharges with low $\mathrm{D}_{2}$ gas rates, while, at high gas rates and high $\beta$, pre-ELM pedestals are found to be stable to P-B modes, although type I ELMs occur experimentally [1]. Note that, high $\mathrm{D}_{2}$ gas rates are required in JET-ILW to increase the ELM frequency in order to achieve core tungsten control over longer time scales [12]. Furthermore, at high gas rates, the inter-ELM temperature pedestal growth is saturated half way through the ELM cycle leading to plasmas with reduced confinement [2].

Saturation of the temperature and its gradient prior to the ELM crash has also been observed on ASDEX-Upgrade [13, 14], DIII-D [15] and on C-mod [16], although the dependence of the saturation on the fuelling gas rate is not discussed in these studies. These reports have also found that the recovery time of the density pedestal is generally shorter than that of the temperature pedestal. Furthermore, the saturation of the temperature gradient is correlated with the onset of quasi-coherent fluctuations, implying that these fluctuations may play a role in regulating the edge transport. Possible explanations for the delay of the ELM crash when the pedestal evolution is saturated are also considered. The report on DIII-D pedestals [15] argues that quasi- 
coherent fluctuations limit and saturate the pedestal gradient, but allow the width and height to possibly further increase. However, experimental data where both the density and temperature heights and gradients are saturated is also presented, leaving the question of the ELM crash delay open. A recent study on ASDEX-Upgrade pedestals shows that the total stored energy increases while the pedestal parameters are saturated, possibly causing stabilisation of P-B modes and delaying the ELM crash [14]. A time lag in the build-up of the total edge current with respect to the pressure gradient due to current diffusion could also be responsible for the delay of the ELM crash. This possibility has been investigated on ASDEX-Upgrade $[13,17]$, showing that the current diffusion only plays a minor role in the edge of ASDEX-Upgrade H-mode plasmas.

In order to try and understand the origin of the contradiction between the pedestals stable to P-B modes and the experimentally observed type I ELMs on JET-ILW, in this paper we study the time evolution of the edge current during the ELM cycle, investigating both the edge bootstrap current density profile $\left(j_{\mathrm{BS}}\right)$ and the Ohmic component $\left(j_{\mathrm{OH}}\right)$. A similar approach is taken as in $[13,17]$ for ASDEX-Upgrade, but this investigation accounts for pedestals at higher temperature, where potentially the effect of current diffusion is more dominant due to the higher plasma conductivity.

The measurement of the edge current density is very challenging, and to date there is no available diagnostic for this purpose on JET. Thus, in this paper we calculate the bootstrap current with the local neoclassical code NEO [18, 19], and we account for the Ohmic contribution to the total current, by solving the current diffusion equation in the plasma. For this analysis the JETTO [20] transport code has been utilised to consider a realistic geometry. Consecutive ELM cycles are simulated in order to examine the effect of current diffusion on the inter-ELM edge current profile evolution in circumstances where any transients introduced by the initial condition are relaxed.

The paper is organised as follows. In section 2 we describe the method used to calculate the edge bootstrap current in JET-ILW H-modes. The inter-ELM evolution of the edge bootstrap current is studied in section 3. Section 4 analyses the effect of current diffusion on the evolution of the edge current inter-ELM for a range of JET-ILW pedestal collisionalities. Summary and conclusions are presented in section 5 .

\section{Edge bootstrap current density profile}

In the steep pressure gradient region at the edge of H-mode plasmas, the current density is usually dominated by the bootstrap current. In the present section only the bootstrap current is considered and the effect of the Ohmic current on the inter-ELM evolution of the total edge current is discussed in section 4. The bootstrap current is parallel to the magnetic field and is driven by the radial pressure gradient. As the bootstrap current is associated with the existence of trapped particles, it strongly depends on the collisionality. Trapped particles which are scattered into passing orbits before completing their banana orbits do not contribute to the bootstrap current. 
In this paper the bootstrap current density is computed from first principles with the local neoclassical transport code NEO [18, 19], which solves the drift-kinetic equation with a full linearized Fokker-Planck collision operator including all inter-species collisions. This approach provides a more accurate estimation of the bootstrap current than the widely used Sauter-formula [21, 22], especially at high collisionality, where the Sauter-formula has been shown to overestimate the bootstrap current by up to $100 \%$ compared to NEO for JET-ILW pedestals [1]. The parallel bootstrap current density $\left(j_{\mathrm{BS}}\right)$ and the parallel component of the total current density $\left(j_{\text {tot }}\right)$ profiles in this paper are expressed in the form of a flux surface average:

$$
j_{\mathrm{BS}}=\frac{\left\langle\boldsymbol{j}_{\mathrm{BS}} \cdot \boldsymbol{B}\right\rangle}{B_{\mathrm{ax}}}, j_{\mathrm{tot}}=\frac{\left\langle\boldsymbol{j}_{\mathrm{tot}} \cdot \boldsymbol{B}\right\rangle}{B_{\mathrm{ax}}},
$$

where $B$ is the magnetic field and $B_{\text {ax }}$ is the magnetic field on axis.

The inputs for NEO are the EFIT plasma equilibrium, the electron $\left(T_{e}\right)$ and ion $\left(T_{i}\right)$ temperatures and the electron $\left(n_{e}\right)$ and ion density $\left(n_{i}\right)$ profiles. The kinetic profiles are obtained by fitting the electron kinetic profiles from the high resolution Thomson scattering (HRTS) measurements, assuming equal electron and ion temperatures (consistent with charge exchange measurements at the pedestal top), constant line averaged $Z_{\text {eff }}$ measured by Visible Bremsstrahlung and Be as the intrinsic impurity.

The HRTS profiles collected from a steady time window of the discharge are ELMsynchronised to improve signal statistics [23, 24]. In order to track the time dependent evolution of $j_{\mathrm{BS}}$ during the ELM cycle, the ELM cycle is divided into $20 \%$ long intervals, as shown in figure 1a. $100 \%$ corresponds to the onset of the ELM crash and $0 \%$ corresponds to the ELM crash of the preceding ELM cycle. Thus, the 0-20\% interval is excluded from the analysis as the profiles in this interval are often dominated by the particular dynamics of each ELM crash. Composite profiles are taken from each $20 \%$ bin and a modified tanh function [25] is fitted to both the electron temperature and density profiles.
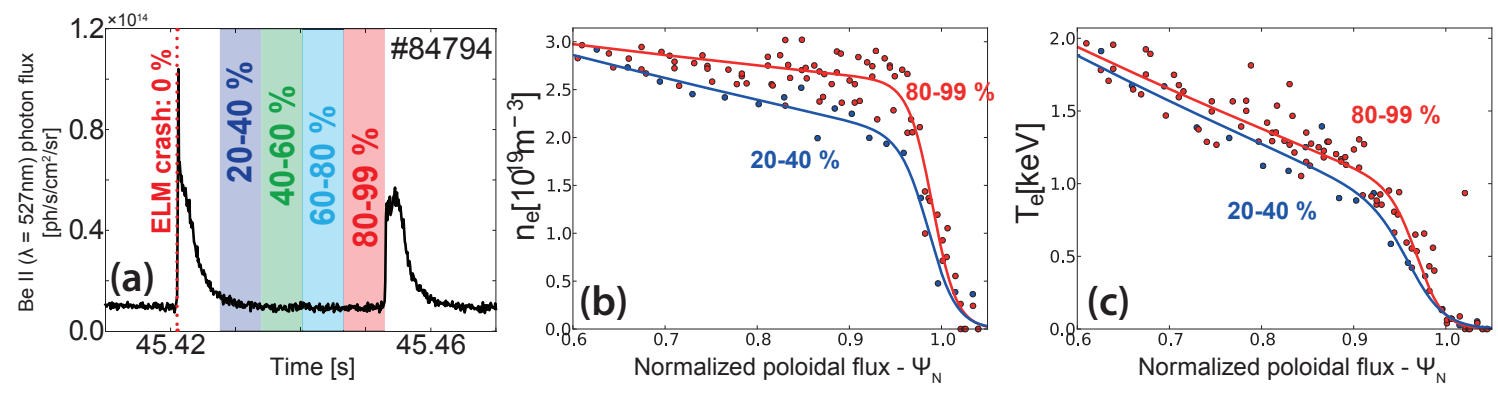

Figure 1. (a) The ELM cycle is normalised to a relative time scale (from $0 \%$ to 100 $\%)$ and divided into $20 \%$ long intervals. The ELM marker is the Be II $(\lambda=527 \mathrm{~nm})$ photon flux measured at the inner divertor. (b) and (c) The $n_{e}$ and $T_{e}$ experimental data for the 20-40\% and 80-99\% intervals in \#84794. 
Examples of fitted $n_{e}$ and $T_{e}$ profiles derived from composite HRTS measurements are shown in figure 2a and 2b, respectively, for JET-ILW discharge \#84794 (1.4 MA/1.7 $\mathrm{T}$, input power $P_{\mathrm{IN}} \simeq 16 \mathrm{MW}$, average triangularity $\delta=0.27$, injected gas rate $\Gamma_{D}=2.8 \cdot 10^{21} \mathrm{e} / \mathrm{s}$ ) for the four inter-ELM intervals from $20-40 \%$ to $80-99 \%$. The $n_{e}$ and $T_{e}$ experimental data for the 20-40\% and 80-99\% intervals in figure $1 \mathrm{~b}$ and c show that the observed difference in the fitted profiles is beyond any experimental uncertainty, as seen in the scatter in the data points. Figure 2c shows the inter-ELM evolution of the edge electron pressure gradient. In this discharge the peak pressure gradient continuously increases during the inter-ELM cycle as typically observed at low gas rates. The inter-ELM evolution of the $j_{\mathrm{BS}}$ profile is shown in figure $2 \mathrm{~d}$, showing a similar time evolution to that of the pressure gradient, as expected.
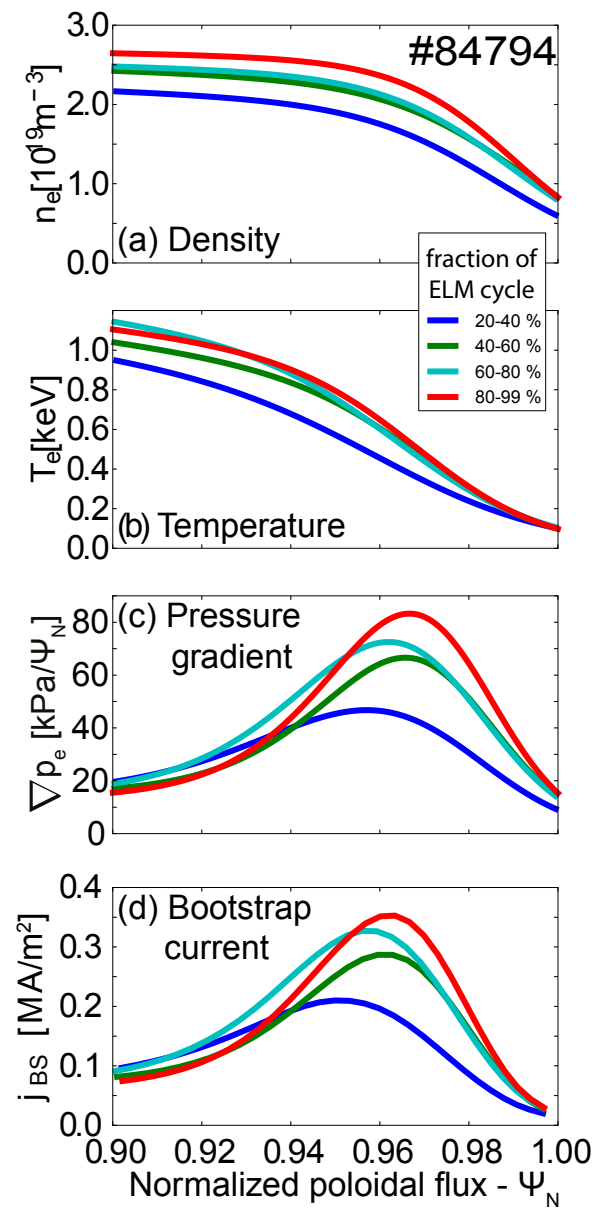

Figure 2. Time evolution of (a) the edge electron density $\left(n_{e}\right)$; (b) the edge electron temperature $\left(T_{e}\right) ;(\mathrm{c})$ the edge electron pressure gradient $\left(\nabla p_{e}\right)$ and $(\mathrm{d})$ the edge bootstrap current density $\left(j_{B S}\right)$ profiles in the inter-ELM cycle of pulse \#84794, evaluated for the four intervals: $20-40 \%, 40-60 \%, 60-80 \%, 80-99 \%$ of the total ELM cycle.

The sensitivity of the $j_{B S}$ profiles to the uncertainties of the input parameters $n_{e}$, $T_{e}$ and $Z_{\text {eff }}$, has been investigated. For this purpose, "modulated" $n_{e}$ and $T_{e}$ profiles were constructed, whereby each fitted parameter of the mtanh function (width, height, 
position and slope) was substituted with a Gaussian random number. The mean and variance of the Gaussian random numbers are given by the mean and variance of the parameter estimates of the mtanh fit. For each case, the $j_{B S}$ profile calculation with NEO is then repeated several times $(\sim 50)$ using as input the different "modulated" profiles. The line averaged $Z_{\text {eff }}$ is varied within the experimental uncertainty of $\pm 10 \%$. The result of the sensitivity analysis is shown in figure 3, where all three parameters $\left(n_{e}, T_{e}, Z_{\text {eff }}\right)$ are modulated within their uncertainties. The percentile of the resulting $j_{B S}$ profiles gives the $1 \sigma_{s d}$ and $2 \sigma_{s d}$ error bars where $\sigma_{s d}$ is the standard deviation. The error bars are visible in figure 3 in red and blue, respectively. Since all three parameters $\left(n_{e}, T_{e}, Z_{\text {eff }}\right)$ were assumed to be independent in the uncertainty analysis, the resulting error bars are somewhat overestimating the error. Systematic errors were not taken into account in this analysis.

Tests where only one input parameter is "modulated" have shown that the uncertainty in the calculated $j_{B S}$ is dominated by the uncertainty in the $T_{e}$ profiles. This is due to the fitted HRTS $T_{e}$ profiles having a larger uncertainty than the $n_{e}$ profiles in the JET-ILW dataset analysed in this study. In the remainder of the paper the $1 \sigma_{s d}$ error bars are used as the uncertainty in the $j_{\mathrm{BS}}$ profile.

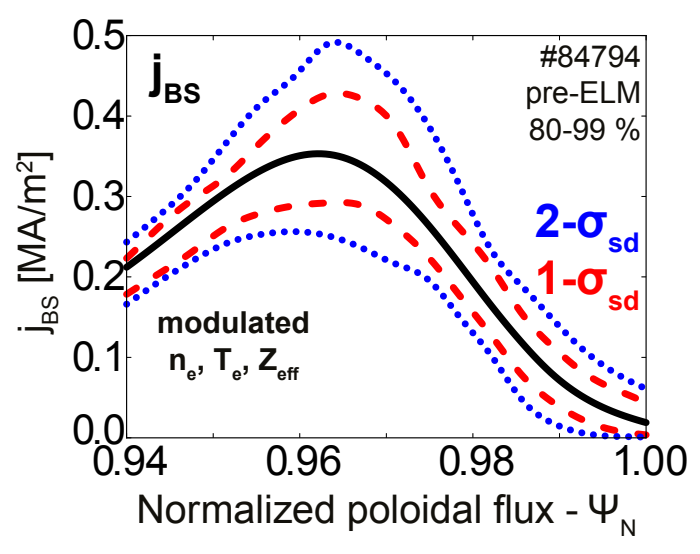

Figure 3. Edge $j_{\text {BS }}$ profile for pulse \#84794 in the last $20 \%$ of the ELM cycle (black) and $1-\sigma_{s d}$ (red) and $2-\sigma_{s d}$ (blue) uncertainties, derived from the sensitivity analysis on the $j_{\mathrm{BS}}$ calculation to $n_{e}, T_{e}$ and $Z_{\mathrm{eff}}$ uncertainties.

\section{Inter-ELM evolution of the edge bootstrap current}

In JET-ILW type I ELMy H-modes at low $D_{2}$ gas rates the edge pressure gradient continuously increases during the inter-ELM phase, until the ELM crash [2]. On the other hand, at high $D_{2}$ gas fuelling rates and at high $\beta$, the pressure edge gradient is typically saturated in the second half of the ELM cycle and the pre-ELM pedestals have been shown to be stable to P-B modes $[1,2]$. In the latter cases, the global confinement $\left(H_{98(y, 2)}\right)$ is lower than in pulses with low gas rates at the same input power, primarily due to lower temperature pedestals. In this section the inter-ELM evolution of the edge 
bootstrap current is investigated and compared to the time evolution of the density, temperature and pressure gradients in the ELM cycle.

We consider the set of type I ELMy H-mode discharges studied in $[1,2]$. The plasmas were at $1.4 \mathrm{MA} / 1.7 \mathrm{~T}$, low triangularity $(\delta), P_{\text {sep }}=4 \rightarrow 14 \mathrm{MW}$ (where $P_{\text {sep }}$ is the power across the separatrix $\left.P_{\text {sep }}=P_{\mathrm{IN}}-\mathrm{d} W / \mathrm{d} t-P_{\text {rad,bulk }}\right)$, gas rate: $\Gamma_{\mathrm{D}_{2}}=2.8 \rightarrow 18 \cdot 10^{21} \mathrm{e} / \mathrm{s}$, normalised $\beta: \beta_{N}=1.2 \rightarrow 2.8$ and ELM frequency: $f_{\mathrm{ELM}}=12 \rightarrow 120 \mathrm{~Hz}$. In the experiment, at a given $P_{\text {sep }}$, the electron pressure at the pedestal top $\left(p_{e, P E D}[\mathrm{~Pa}]=1.602 \times n_{e, P E D}\left[10^{-19} \mathrm{~m}^{-3}\right] \times T_{e, P E D}[\mathrm{eV}]\right.$, where 1.602 originates from the elementary charge as $T_{e}$ is measured in $\mathrm{eV}$ ) is reduced with increasing gas rate mainly via a reduction in the pedestal temperature. The pedestal collisionality $\left(\nu_{e, P E D}\right)$ is mainly driven by the variation in the pedestal temperature in the power and gas scans [1] and varies between $\nu_{e, P E D}=0.3 \rightarrow 4.5$. Electron-ion collisionalities $\left(\nu_{e}\right)$ presented in this paper are calculated using eq. (18b) in [21].

Figure 4 compares the inter-ELM evolution of $j_{\mathrm{BS}}$ at different gas rates: at low $P_{\text {sep }}=4 \mathrm{MW}(4 \mathrm{a})$ and at high $P_{\text {sep }}=13 \mathrm{MW}(4 \mathrm{~b}) . \quad j_{B S}$ is reduced at low power due to the lower $T_{e}$ and thus higher $\nu *$ compared to the high $P_{\text {sep }}$ case. At "low" gas injection (where the pre-ELM stability is consistent with the P-B model [1]), the peak $j_{B S}$ continuously increases during the ELM cycle. This change in peak $j_{B S}$ is beyond the uncertainties indicated with the $1 \sigma$ error bars. Conversely, at "medium" and "high" gas rates the evolution of peak $j_{B S}$ is roughly constant throughout the second half of the ELM cycle within the $1 \sigma$ error bars. This saturation of peak $j_{\mathrm{BS}}$ during the ELM cycle at high gas rate is observed both at low and high input power.
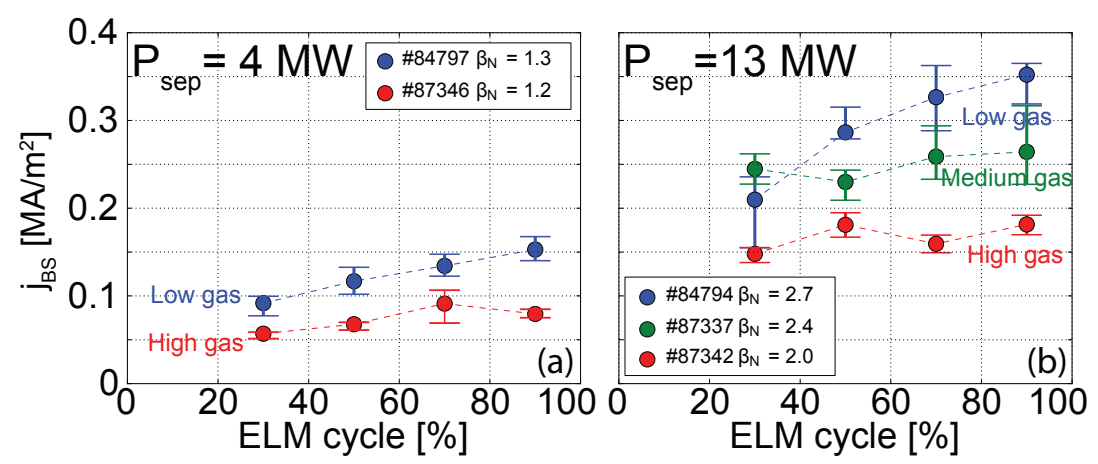

Figure 4. Evolution of peak $j_{B S}$ during the ELM cycle in type I ELMy H-modes at low $\left(2.8 \cdot 10^{21} \mathrm{e} / \mathrm{s}\right)$, medium $\left(8.4 \cdot 10^{21} \mathrm{e} / \mathrm{s}\right)$ and high $\left(18 \cdot 10^{21} \mathrm{e} / \mathrm{s}\right)$ gas rates at constant $P_{\text {sep }}$ : (a) lowest power and (b) highest power of the power and gas scans dataset.

It is interesting to compare the inter-ELM $j_{B S}$ evolution to that of the separate drives of $\nabla p_{e}$, namely the temperature and density gradients, as shown in figure 5 , where all values are normalised to the pre-ELM phase value. Figure 5 shows the evolution of the peak of the edge $j_{B S}$ profile and the peak of the gradients, thus the radial location the data corresponds to is changing in time. For all shots of the dataset, we observe that peak $j_{B S}$, peak $\nabla p_{e}$ and peak $\nabla T_{e}$ always exhibit the same time evolution, regardless of the $\nabla n_{e}$ inter-ELM evolution. Figure 5a shows the case of pulse \#84794 where 
the pedestal $\nu^{*}$ is lowest in the scan: peak $\nabla n_{e}$, peak $\nabla T_{e}$ and peak $\nabla p_{e}$ all increase during the ELM cycle. In figure $5 \mathrm{~b}$ a higher $\nu^{*}$ case (\#87342) is shown, where the evolution of peak $\nabla p_{e}$ is saturated and closely follows the evolution of peak $\nabla T_{e}$, but the evolution peak $\nabla n_{e}$ is different, with $\nabla n_{e}$ first increasing and then decreasing. This latter behaviour is typical of the whole dataset, suggesting that the evolution of peak $\nabla p_{e}$ is driven by the time evolution of peak $\nabla T_{e}$.
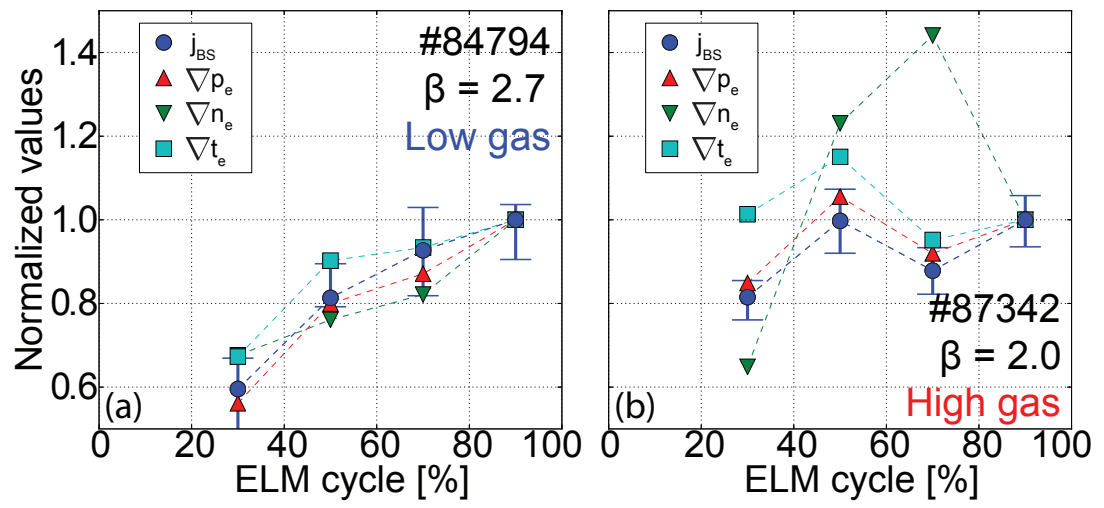

Figure 5. Inter-ELM evolution of peak $j_{B S}$ and peak of $p_{e}, n_{e}, T_{e}$ gradients at low and high gas rates at $P_{\mathrm{sep}}=13 \mathrm{MW}$. All values are normalised to the pre-ELM phase. The error bars are shown only for peak $j_{B S}$ to avoid overcrowding of the plot, but all data points have error bars of magnitude similar to those shown.

The dominant effect of the temperature gradient on $j_{\mathrm{BS}}$ can be understood by separately evaluating the $\nabla n_{e}$ and $\nabla T_{e}$ terms of $\nabla p_{e} \sim n_{e} \nabla T_{e}+T_{e} \nabla n_{e}$. In JETILW pedestals, the maximum $\nabla T_{e}$ is typically located radially inwards of the maximum $\nabla n_{e}[26,27]$, as can be seen e.g. in figure 2 for pulse \#84794. This relative radial shift between $T_{e}$ and $n_{e}$ gradients leads to a smaller contribution of the $T_{e} \nabla n_{e}$ term to $\nabla p_{e}$, as the temperature is low where the density gradient peaks, explaining why the pressure gradient is dominated by the $n_{e} \nabla T_{e}$ term. The ratio of $n_{e} \nabla T_{e} / T_{e} \nabla n_{e}$ is $\sim 4$ in the dataset at the maximum $\nabla p_{e}$.

For the study of the separate contributions from $\nabla n_{e}, \nabla T_{e}$ and $\nabla T_{i}$ to the bootstrap current Sauter's analytical formula $[21,22]$ is used instead of NEO. The NEO code solves the drift-kinetic equation and its output is the particle distribution function, thus it is not straightforward to extract information on the separate drives of the density and temperature gradients. The Sauter formula is an analytical fit to the results of neoclassical codes and it expresses the bootstrap current as a function of density and temperature gradients, collisionality and parameters of the magnetic equilibrium. Due to the approximate electron-ion collision operator used in the simulation and also due to the simplified fitting formula used at high collision frequency it is less accurate at higher electron collisionality [28]. However, it is assumed that it gives a good estimation for the ratios between the different drives of $j_{\mathrm{BS}}$, especially at low collisionalities where it has been shown to be in good agreement with the NEO results. The coefficients of 
Sauter's formula were evaluated in the investigated pedestals:

$$
\begin{aligned}
j_{\mathrm{BS}}^{\text {Sauter }}= & \frac{I(\Psi) p(\Psi)}{B_{\mathrm{ax}}}[\underbrace{\mathcal{L}_{31}}_{\text {coeff. of } \nabla n_{e}} \frac{\partial \ln n_{e}}{\partial \Psi}+\underbrace{R_{p e}\left(\mathcal{L}_{31}+\mathcal{L}_{32}\right)}_{\text {coeff. of } \nabla T_{e}} \frac{\partial \ln T_{e}}{\partial \Psi}+ \\
& +\underbrace{\left(1-R_{p e}\right)\left(1+\frac{\mathcal{L}_{34}}{\mathcal{L}_{31}} \alpha\right) \mathcal{L}_{31}}_{\text {coeff. of } \nabla T_{i}} \frac{\partial \ln T_{i}}{\partial \Psi}],
\end{aligned}
$$

where $I(\Psi)=R B_{t}, R_{p e}=p_{e} / p$ and $\alpha, \mathcal{L}_{31}, \mathcal{L}_{32}, \mathcal{L}_{34}$ are coefficients which can be analytically computed from equations (13) through (18) in [21]. The coefficient of $\nabla n_{e}$ is larger than the coefficient of $\nabla T_{e}$ and $\nabla T_{i}$ in the range of pedestal collisionalities considered in this paper. Despite the higher coefficient for $\nabla n_{e}$, the bootstrap current is dominated by the $\nabla T_{e}$ term of Sauter's formula. The ratio of $n_{e}, T_{e}$ and $T_{i}$ drives is approximately 1:3:1 in the dataset at the maximum $\nabla p_{e}$. This result also implies that (in case of local calculation of the bootstrap current) the error introduced by the $T_{e}=T_{i} \Rightarrow \nabla T_{e}=\nabla T_{i}$ approximation is not significant as the contribution from $\nabla T_{i}$ to the bootstrap current is small.

\section{Effect of Ohmic current diffusion on the total edge current}

JET-ILW pre-ELM pedestals at high gas rates and high $\beta$ are stable to P-B modes, indicating that additional physics may be required to explain the ELM trigger [1]. Furthermore, in high $\beta$, high gas rate discharges both the pressure gradient $[1,2]$ and the peak bootstrap current (see section 3) reach their steepest gradient well before the ELM crash. Therefore, a possible explanation for the "delay" in the ELM crash could be a time delay in the build up of the total edge current inter-ELM due to current diffusion with respect to the inter-ELM recovery of density and temperature gradients (which drive the bootstrap current recovery).

The bootstrap current profiles presented in section 3 are calculated from the measured kinetic profiles assuming steady-state conditions. As a result, the time evolution of $j_{\mathrm{BS}}$ follows that of the pressure gradient. The effect of inter-ELM current diffusion can be assessed by taking into account the contribution of the Ohmic current. In [13] and [17], a simple model for current diffusion is used, which helps to understand the dynamics of the edge current in the inter-ELM period. The model includes Ohm's law:

$$
j_{\mathrm{tot}}=\sigma E+j_{\mathrm{BS}},
$$

where $E$ is the electric field and $\sigma$ the plasma conductivity. In general, the second term in eq. (3) includes any non-inductively driven current. Here, only the bootstrap current is considered. Substituting Faraday's law into eq. (3) gives a current diffusion equation:

$$
\frac{\partial E}{\partial t}=\frac{1}{\sigma}\left(\frac{\nabla^{2} E}{\mu_{0}}-E \frac{\partial \sigma}{\partial t}-\frac{\partial j_{\mathrm{BS}}}{\partial t}\right)
$$

where $\mu_{0}$ is the vacuum permeability and $\partial / \partial t$ is the time derivative. Eq. (4) shows that an increase in bootstrap current $\left(\partial j_{\mathrm{BS}} / \partial t\right)$ reduces the electric field in the pedestal 
build-up phase, such that the electric field opposes the build-up of the total current. Any change in the electric field relaxes in a diffusive process $\left(\nabla^{2} E / \mu_{0} / \sigma\right)$ on the resistive timescale, which is proportional to the plasma conductivity.

In order to solve the current diffusion equation in realistic geometry, JETTO [20] simulations were run within the JINTRAC framework [29]. JINTRAC is a set of linked codes for the integrated simulation of all phases of a tokamak scenario, but here only the JETTO part of the framework was used. The energy and particle transport were not simulated in the JETTO runs: the simulations were run in predictive mode for the current, and in interpretative mode for density and temperature. Since NEO is not implemented in JETTO for the calculation of the bootstrap current, the neoclassical code NCLASS [30] is used for this purpose. The difference between NEO and NCLASS calculated bootstrap currents is less than $10 \%$ in the investigated cases.

The input kinetic profiles for the JETTO runs are the same as those used for the NEO calculations (see section 2). However, in order to enable JETTO to simulate the inter-ELM evolution of the total current, the inter-ELM profiles have to be interpolated on a finer time grid. In particular, the collapse of the profiles during the ELM crash have to be included. Due to the relatively slow time resolution of the HRTS diagnostic (20 ms), the short time scale of the ELM crash $(\leq \mathrm{ms})$ cannot be resolved. Thus, the temperature and density profile evolution during the ELM-crash is modelled by a simple, linear interpolation in time between the pre-ELM and the post-ELM profiles. In order to obtain a more realistic picture of the sudden change of the pedestal profiles at the ELM crash, the timescale of the crash is estimated from the interferometry and electron cyclotron emission (ECE) measurements, both equipped with higher time resolution than HRTS ( $<1 \mathrm{~ms}$ for ECE and $1.5 \mathrm{~ms}$ for interferometry). An ECE channel located close to the pedestal top and an interferometry line-of-sight through the plasma edge are chosen. The duration of the ELM crash is evaluated by taking the time difference between the maximum and minimum of these signals in the vicinity of the ELM crash. ECE and interferometry are only used for this purpose, while the shape of the density and temperature profiles rely solely on the HRTS measurements.

The effect of any long time-scale evolution in the plasma is eliminated by running the JETTO simulations with steady (pre-ELM) profiles for 5 seconds. Following this period, 10 consecutive ELM cycles are simulated. This approach ensures that the simulation reaches a dynamic equilibrium in which the ELM cycles become identical. Figure 6 illustrates the evolution of the electron pressure close to the pedestal top in the JETTO simulations. In what follows, the results shown belong to the evolution of the last ELM cycle of the sequence.

The fixed boundary equilibrium code ESCO [20] is used to calculate the equilibrium by taking into account the steep pressure gradient at the plasma edge. The plasma boundary is taken from EFIT. ESCO is run only at the very beginning of the simulation to provide the grid on which the current diffusion equation is solved. In the later stage of the simulation, the equilibrium is not self-consistently recalculated, only the current density and $q$ profiles are evolved according to the redistribution of the current. 


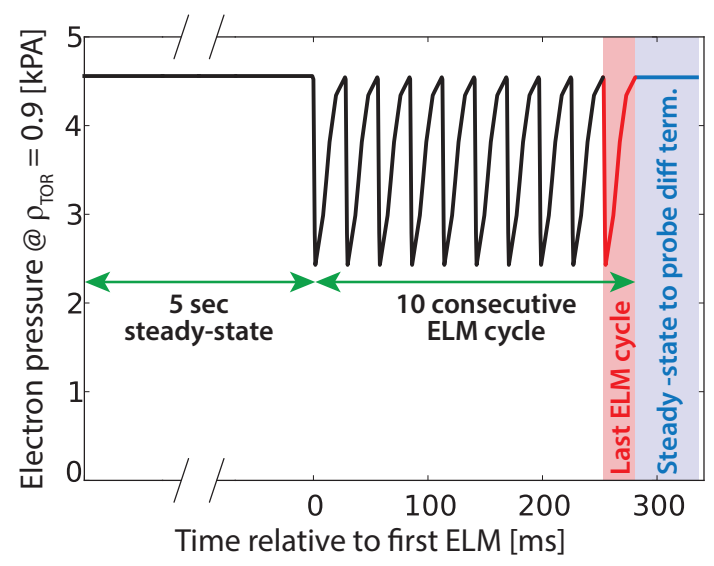

Figure 6. Evolution of the electron pressure close to the pedestal top $\left(\rho_{\mathrm{TOR}}=0.9\right)$ in the JETTO simulations. After 5 seconds of simulation time with steady kinetic profiles, 10 consecutive ELM cycles are simulated. In what follows, the results shown belong to the evolution of the last ELM cycle of the sequence, indicated with the red shaded area. Some simulations were continued with fixed pre-ELM profiles to test the diffusion term in eq. (4). This is indicated with the blue shaded area.

The boundary condition at the separatrix is a key element in the simulation. However, it is challenging to determine the boundary condition experimentally on the time scale of the ELM cycle, as the magnetic measurements are affected by slow data acquisition and the screening of the vacuum vessel and other conducting structures. In order to examine the effect of the choice of boundary condition on the edge current density evolution, two options are tested here:

(i) the total plasma current is held constant,

(ii) the loop voltage is held constant.

It is expected that current diffusion could have a significant effect on the total current build-up if the resistive timescale is comparable to the inter-ELM period. Thus, in section 4.1 two extreme cases of JET-ILW pedestals at low $I_{p}(1.4 \mathrm{MA})$ are investigated in detail: in the first case (pulse \#84794), the pedestal collisionality is $\nu_{\mathrm{e}, \mathrm{PED}}^{*} \simeq 0.3$, the ELM frequency is $f_{\mathrm{ELM}} \simeq 35 \mathrm{~Hz}$ and the pedestal temperature is $T_{\mathrm{e}, \mathrm{PED}} \simeq 0.9 \mathrm{keV}$, thus the conductivity is high and the resistive timescale is longer; the second case is a higher collisionality $\left(\nu_{\mathrm{e}, \mathrm{PED}}^{*} \simeq 0.9\right)$ pulse $(\# 87342)$ with the highest power in the high gas rate scan, with a colder pedestal, $T_{\mathrm{e}, \mathrm{PED}} \simeq 0.6 \mathrm{keV}$, but much higher ELM frequency, $f_{\mathrm{ELM}} \simeq 120 \mathrm{~Hz}$, thus the ELM period may be short enough to be comparable to the resistive timescale. A connection to high $I_{p}$ JET plasmas are achieved by analysing a baseline scenario pedestal obtained at $I_{p}=3.0 \mathrm{MA}$. This is presented in section 4.2, together with sensitivity calculations on the ELM duration and magnitude. Section 4.3 discusses the impact of current diffusion on pedestal stability analyses. 


\subsection{Comparison of pedestals at different fuelling gas rates}

First, the simulation results of the highest pedestal temperature discharge (\#84794, high power, low gas rate) are presented. In figure 7 the inter-ELM evolution of the edge total and bootstrap current profiles are shown, comparing the impact of the two different boundary conditions on the current evolution. Figure $7 \mathrm{a}$ shows the result of the simulation where the total current is held constant at the experimental value: $I_{\mathrm{p}}=1.4 \mathrm{MA}$. Figure $7 \mathrm{~b}$ shows the result of the JETTO run with a fixed loop voltage at the separatrix. $U_{\text {loop }}=0.11 \mathrm{~V}$ is chosen, so that the total plasma current on average is close to 1.4 MA. As a consequence of the flattened kinetic profiles during the ELM crash, the bootstrap current profile (dashed lines in figure 7) drops in the first few ms of the ELM cycle. However, the electric field significantly increases as a response to the bootstrap current drop, mitigating the reduction in the peak total current (solid lines in figure 7). This is visible in figure 8 , where the time evolution of the parallel electric field is shown. Note that the profiles corresponding to $0 \%$ (ELM onset) and $100 \%$ (ELM onset of the subsequent ELM) are almost identical both in figure 7 and 8 . This confirms that any long time-scale evolution of the electric field is relaxed in the simulation and the ELM cycle can be examined in steady-state.
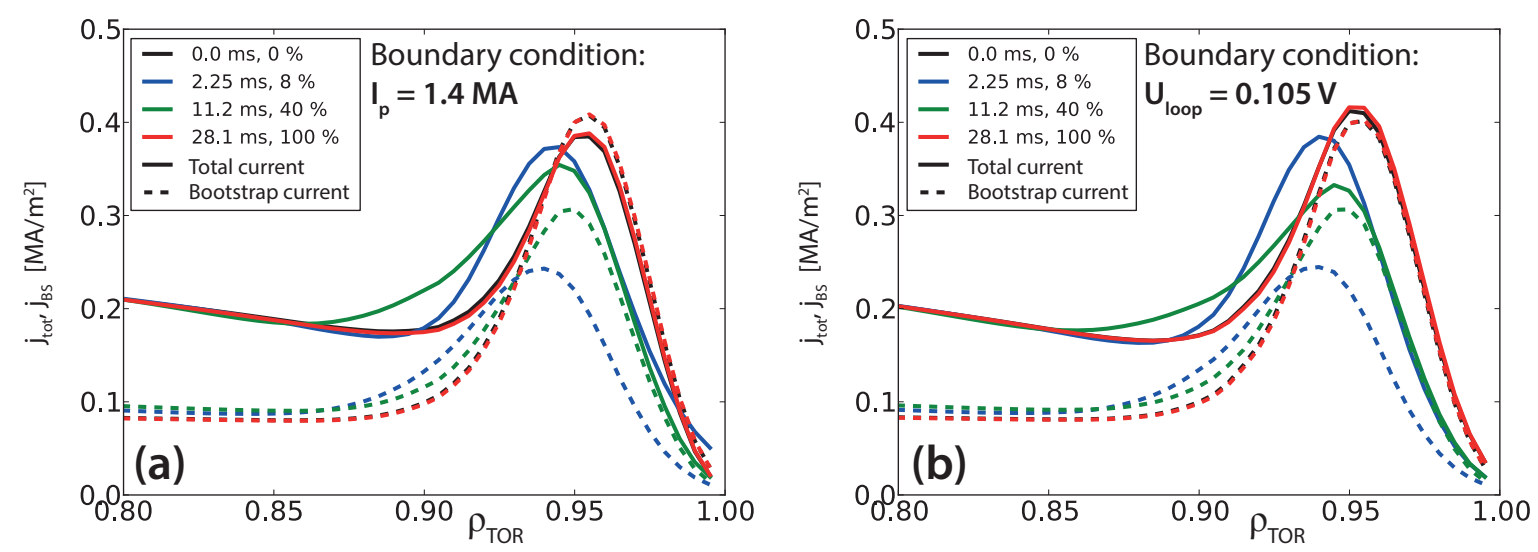

Figure 7. Inter-ELM evolution of the total (solid curves) and bootstrap current (dashed curves) profiles in the plasma edge for pulse \#84794 $\left(T_{\mathrm{e}, \mathrm{PED}}=0.9 \mathrm{keV}\right)$. (a) The total current, (b) the loop voltage held constant as a boundary condition. The bootstrap current profile drops during the ELM crash, but the total current decreases on a slower time scale due to the increase in the electric field (see figure 8).

The inter-ELM evolution of the bootstrap and total current profiles with the two different boundary conditions are shown in figure $9 \mathrm{a}$ at a radial coordinate close to the peak value $\left(\rho_{\mathrm{TOR}}=0.95\right)$. In both cases, the $j_{\mathrm{BS}}$ (dashed lines) significantly drops at the ELM crash, while the drop in the total edge current (solid lines) is relatively small and slightly delayed in time. However, in the second half of the ELM cycle, the peak total current builds-up on a similar timescale as the bootstrap current and the pressure gradient (see figure 9b). 

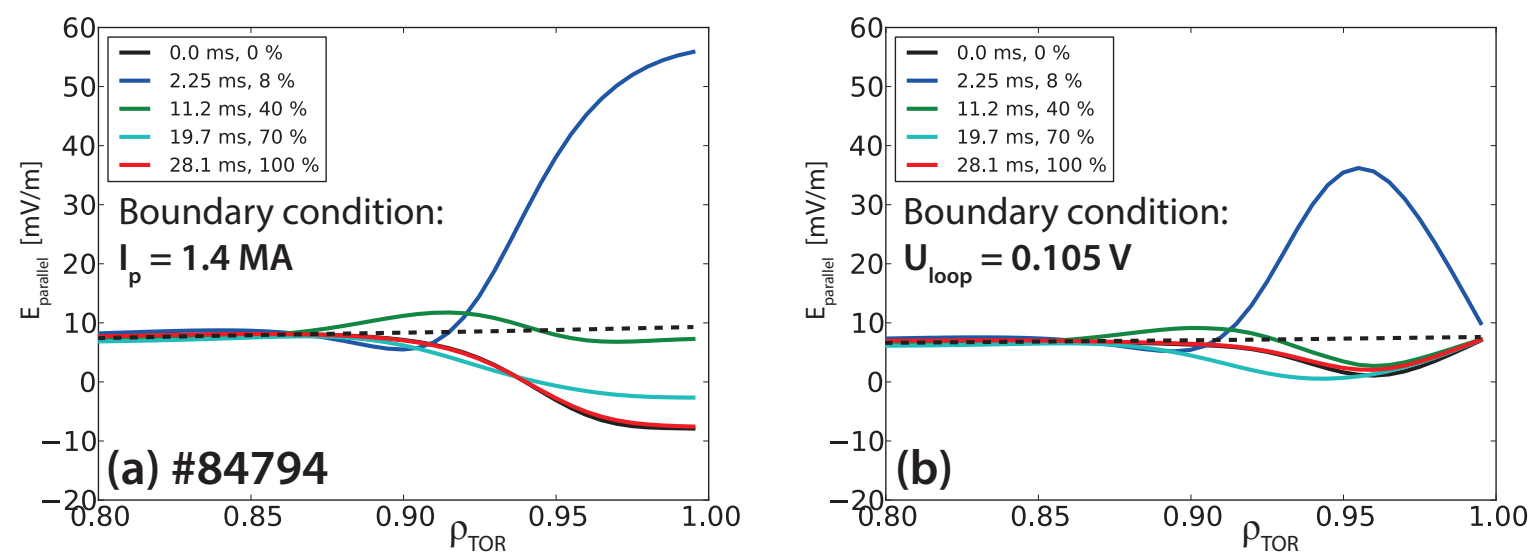

Figure 8. Inter-ELM evolution of the parallel electric field profiles for pulse \#84794 $\left(T_{\text {e,PED }}=0.9 \mathrm{keV}\right)$. (a) Total current held constant as boundary condition, (b) loop voltage at the separatrix held constant as a boundary condition. The electric field significantly increases during the ELM crash (blue curve) as a response to the drop in the edge bootstrap current. The dashed lines show the time-average of the electric field profile over the ELM cycle, which is close to the fully diffused electric field.

The difference between the two simulations can be explained by the effect of the boundary condition on the time evolution of the electric field profile. The time evolution of the loop voltage at the separatrix (which is the boundary of the simulation) is shown in figure $9 \mathrm{c}$. In the $I_{p}=$ const. case, $U_{\text {loop }}^{\text {sep. }}$ is set so that $\partial E / \partial \rho=0$ at the separatrix (see figure 8a), thus the total plasma current is conserved in the system. The prescribed electric field ( $U_{\text {loop }}=2 \pi R_{0} E$ ) at the separatrix also affects the electric field inside the separatrix through the first term on the r.h.s. of eq. (4). This difference is visible in figure 9a: the minimum of the total current is $\sim 20 \%$ lower in the $U_{\text {loop }}^{\text {sep. }}=$ const. case, as some current is lost from the plasma. This can also be seen in figure $9 \mathrm{~d}$ which shows that the total plasma current is reduced after the ELM crash. Furthermore, it is visible in figure $8 \mathrm{~b}$, that $\partial E / \partial \rho<0$ at the plasma edge after the ELM crash, implying that current is lost from the system.

The opposite process occurs in the build-up phase of the ELM cycle, thus the peak total current is higher in the $U_{\text {loop }}^{\text {sep. }}=$ const. case than in the $I_{p}=$ const. simulation prior to the ELM crash. Note that despite having the same input kinetic profiles evolution, the $j_{\mathrm{BS}}$ evolution is slightly different with the two different boundary conditions. This is due to the different total current profile evolution in the two simulations, which affects the $j_{\mathrm{BS}}$ profile through the $q$-profile and the collisionality.

Both simulations indicate that the electric field induced by the second and third terms on the r.h.s. of eq. (4) mitigates the effect of the changing bootstrap current on the total current evolution. As this process is present both at the ELM crash and at the recovery phase of the inter-ELM cycle, it leads to a dynamic equilibrium in which the Ohmic current is redistributed in a way that the peak total current evolution closely follows the build-up of the pressure gradient. 


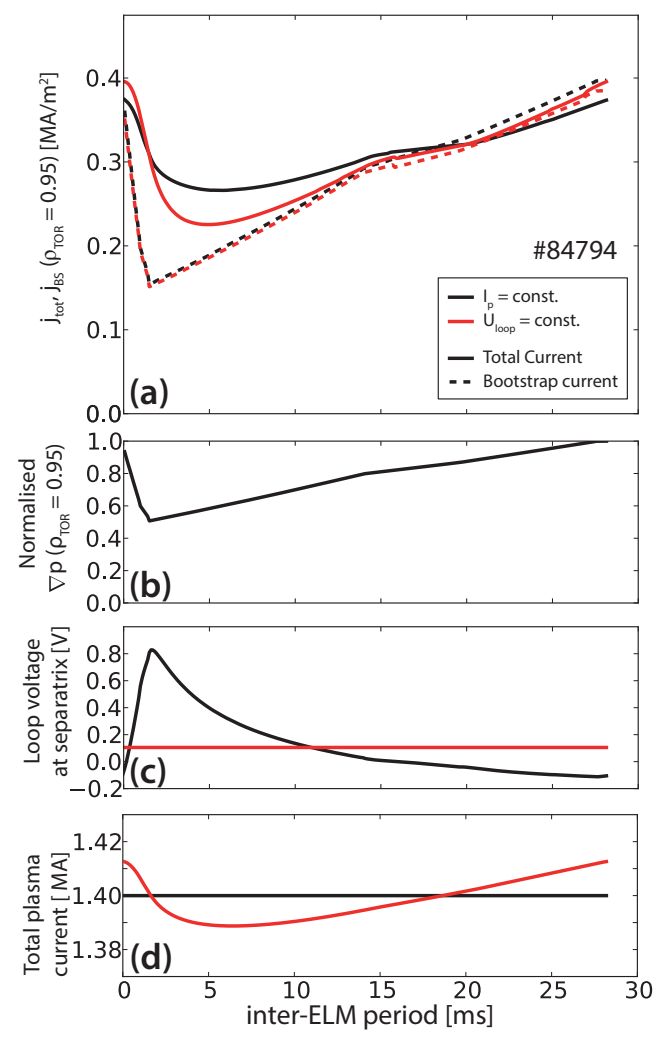

Figure 9. (a) Inter-ELM evolution of the total (solid curves) and bootstrap current (dashed curves) close to the position of peak $j_{\mathrm{BS}}\left(\right.$ at $\left.\rho_{\mathrm{TOR}}=0.95\right)$ in $\# 84794$. The simulation with $I_{p}=$ const. boundary condition is shown in black, and with $U_{\text {loop }}^{\text {sep. }}=$ const. boundary condition in red. The lower panels show the inter-ELM evolution of the: (b) pressure gradient at $\rho_{\mathrm{TOR}}=0.95$ normalised to the maximum, (c) the loop voltage at the separatrix, (d) the total plasma current.

Eq. (4) and the simulation result suggest that the magnitude of the electric field oscillation at the edge during the ELM cycle is proportional to the lost bootstrap current in the ELM crash. The higher the current loss, the larger the induced electric field in the pedestal to keep the total current constant. The opposite applies to the recovery phase of the ELM cycle: the higher the bootstrap current increase, the lower the electric field. Thus, if the ELM magnitude is large (which is likely to lead to large bootstrap current drop through low and/or wide post-ELM crash pedestal), the electric field oscillation is also high. In the JETTO simulation of \#84794 the ELM magnitude is large enough (the ELM energy loss normalised to the pedestal stored energy is $\Delta W_{\mathrm{ELM}} / W_{\mathrm{PED}} \simeq 0.15$ ) that the electric field decreases to negative values, as can be seen in figure 8 . This leads to higher bootstrap current than total current at $\rho_{\mathrm{TOR}}=0.95$ in the pre-ELM phase, as shown in figure $9 \mathrm{a}$.

Figure 9 suggests that the effect of current diffusion on the evolution of the total current in the second half of the ELM cycle is negligible. On the other hand, we note that the electric field profile is not fully diffused by the end of the ELM cycle (see black 
and red curves in figure 8). It is expected that in a simulation with constant profiles (i.e. no ELMs) the electric field reaches a fully diffused state. In the JETTO runs presented in this paper, the time-averaged electric field over the ELM cycle (dashed lines in figure 8) is very close to the fully diffused electric field which would be obtained in a time independent (i.e. no ELMs) simulation. Therefore, in what follows, we refer to this time-averaged electric field as fully diffused. $\ddagger$

In order to understand why the electric field is not fully diffused, but is lower than the time-average by the end of the ELM cycle, all 3 terms on the r.h.s. of eq. (4) and the boundary conditions need to be considered. As mentioned earlier in this section, $U_{\text {loop }}^{\text {sep. }}$ changes in the $I_{p}=$ const. simulation in a way to keep $\partial E / \partial \rho=0$ at the separatrix. It can be seen in figure $8 \mathrm{a}$ that the electric field at the separatrix is significantly lower than the fully diffused value, preventing the electric field profile from reaching the fully diffused state. On the other hand, this constraint does not exist when $U_{\text {loop }}^{\text {sep. }}$ is fixed in the simulation (see figure $8 \mathrm{~b}$ ), but also in this case the electric field profile is still not diffused by the end of the ELM cycle. This is because the third term on the r.h.s. of eq. (4) also plays a role in the inter-ELM evolution of the electric field. This term is inversely proportional to the rate of change of $j_{\mathrm{BS}}$. Since, in pulse \#84794 $j_{\mathrm{BS}}$ increases in the second half of the inter-ELM cycle as shown in figure $9 \mathrm{a}$, the $\partial / \partial t=0$ solution of eq. (4) (assuming a constant, negative $-\partial j_{\mathrm{BS}} / \partial t / \sigma$ term) is smaller than the fully diffused electric field.

It is interesting to evaluate the time scale required for the electric field to reach the fully diffused state, if only the diffusion term was considered in eq. (4). In order to quantify this, the JETTO simulations were continued after the last ELM cycle but with constant pre-ELM profiles, as depicted with the blue shaded area in figure 6 . This simulation represents the evolution of the electric field when only the first term on the r.h.s. of eq. (4) is non-zero, as conductivity and $j_{\mathrm{BS}}$ do not change in time when the kinetic profiles are kept constant. $\S$ Figure 10 shows the evolution of the parallel electric field close to the peak of the total current profile (at $\rho_{\mathrm{TOR}}=0.95$ ). The interELM evolution of $E_{\|}$in the last ELM cycle is shown in red. The rest of the JETTO simulation when the kinetic profiles are kept constant in time and $E_{\|}$relaxes towards the fully diffused state is in blue. It can be seen that the resistive timescale on which the relaxation process takes place is very long $\left(\tau_{\text {res }} \sim 20 \mathrm{~ms}\right)$.

In conclusion, although the resistive time scale in the pedestal is comparable to the ELM period, when the whole ELM cycle (including the ELM crash) is simulated, the resulting time evolution of the peak total current in the second half of the ELM

$\ddagger$ In stationary conditions, the loop voltage $U_{\mathrm{pl}}=\mathrm{d} \Psi / \mathrm{d} t$ evaluated at fixed $\rho_{\mathrm{TOR}}$ is constant in time and flat as function of $\rho_{\mathrm{TOR}}$ if the vacuum magnetic field is constant $\left(\mathrm{d} B_{0} / \mathrm{d} t=0\right.$ is the case in most present day tokamaks) [31]. In figure 8, the flux surface averaged parallel component of the electric field $E_{\|}=\langle E \cdot B\rangle / B_{0}=U_{\|} /(2 \pi R)$ is shown, thus this quantity is not exactly radially constant in steady state conditions. $U_{\mathrm{pl}}$ can be evaluated from $E_{\|}=2 \pi R U_{\|}$in the following way: $U_{\mathrm{pl}}=U_{\|} \mathrm{d} V / \mathrm{d} \rho_{\mathrm{TOR}} /\left(4 \pi^{2} \rho_{\mathrm{TOR}} R_{0}\right)[31]$.

$\S$ Since the current diffusion modifies the total current distribution, there is also a slight change in $j_{\mathrm{BS}}$ through the altered $q$-profile, but this effect is negligible compared to the diffusive process. 


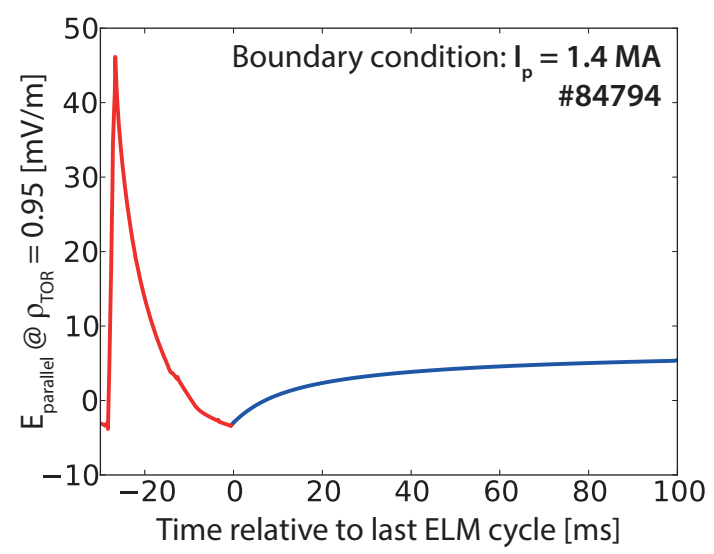

Figure 10. The evolution of the parallel electric field close to the peak of the total current profile (at $\left.\rho_{\mathrm{TOR}}=0.95\right)$ in \#84794. After the last ELM cycle (in red), the simulation continues with the kinetic profiles kept constant in time (in blue).

cycle closely follows that of the bootstrap current and pressure gradient, as shown in figure 9. This is a result of the complex interplay between the different terms of the current diffusion equation (eq. (4)) and the boundary conditions.

Qualitatively, similar conclusions are reached for the JET-ILW pedestal at lower pedestal temperature, but higher ELM frequency $\left(\# 87342, T_{\mathrm{e}, \mathrm{PED}}=0.6 \mathrm{keV}, f_{\mathrm{ELM}} \simeq\right.$ $\left.120 \mathrm{~Hz}, \nu_{\mathrm{e}, \mathrm{PED}} \simeq 0.9\right)$. For this pedestal, the results of the JETTO simulations with $I_{p}=$ const. boundary condition is shown in figure 11. The inter-ELM evolution of the total and bootstrap current at a radius close to the peak $j_{\mathrm{BS}}\left(\rho_{\mathrm{TOR}}=0.95\right)$ is shown in red and at a radius close to the pedestal top $\left(\rho_{\mathrm{TOR}}=0.93\right)$ in black. At $\rho_{\mathrm{TOR}}=0.95, j_{\text {tot }}$ reaches saturation slightly later than $j_{\mathrm{BS}}$ and $\nabla p$. The time lag is less than $1 \mathrm{~ms}$, thus this delay is considered non-significant compared to the inter-ELM period $(\approx 8 \mathrm{~ms})$. The peak $j_{\mathrm{BS}}$ in the second half of the ELM cycle is saturated and the total current evolves similarly, but it slightly decreases towards the end of the ELM cycle. The slight decrease in peak $j_{\mathrm{BS}}$ in this discharge can be understood by taking into account the bootstrap current evolution at the top of the pedestal. Figure 12 shows the inter-ELM evolution of the bootstrap and total current profiles. It is visible that the $j_{\mathrm{BS}}$ profile substantially changes inside the peak of the profile around $\rho_{\mathrm{TOR}}=0.93$. This is also shown in figure 11a with black, where it can be seen that in the second half of the ELM cycle $j_{\mathrm{BS}}$ is roughly constant at $\rho_{\mathrm{TOR}}=0.95$, but it increases at $\rho_{\mathrm{TOR}}=0.93$. This increase in $j_{\mathrm{BS}}$ leads to a decrease in the electric field at $\rho_{\mathrm{TOR}}=0.93$ (see figure $12 \mathrm{~b}$ ), which also affects the electric field evolution at $\rho_{\mathrm{TOR}}=0.95$ through the diffusive term of eq. (4). The evolution of the total current in pulse \#87342 shows that the effect of current diffusion on the time evolution of the total current is complex and it is not sufficient to study the profile evolution at the peak. 


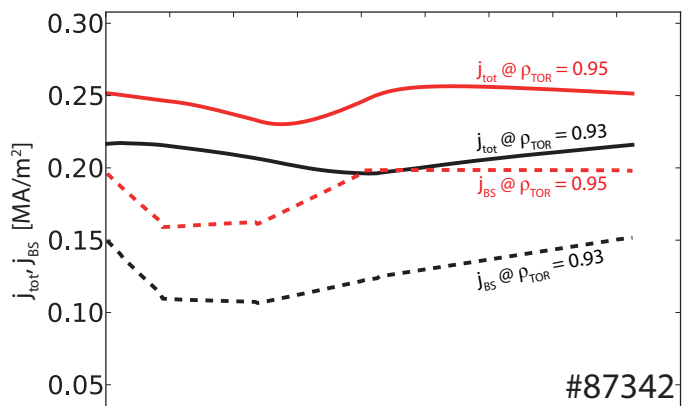

(a) Boundary condition: $I_{p}=1.4 \mathrm{MA}$

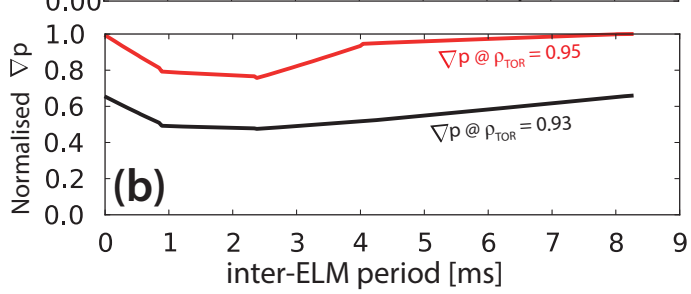

Figure 11. (a) Inter-ELM evolution of the total (solid curves) and bootstrap current (dashed curves) profiles at two radial coordinates close to the peak, $\rho_{\mathrm{TOR}}=0.95$, and close to the pedestal top $\rho_{\mathrm{TOR}}=0.93$ in \#87342 with $I_{p}=$ const. boundary condition. (b) The evolution of the pressure gradient in the inter-ELM cycle.

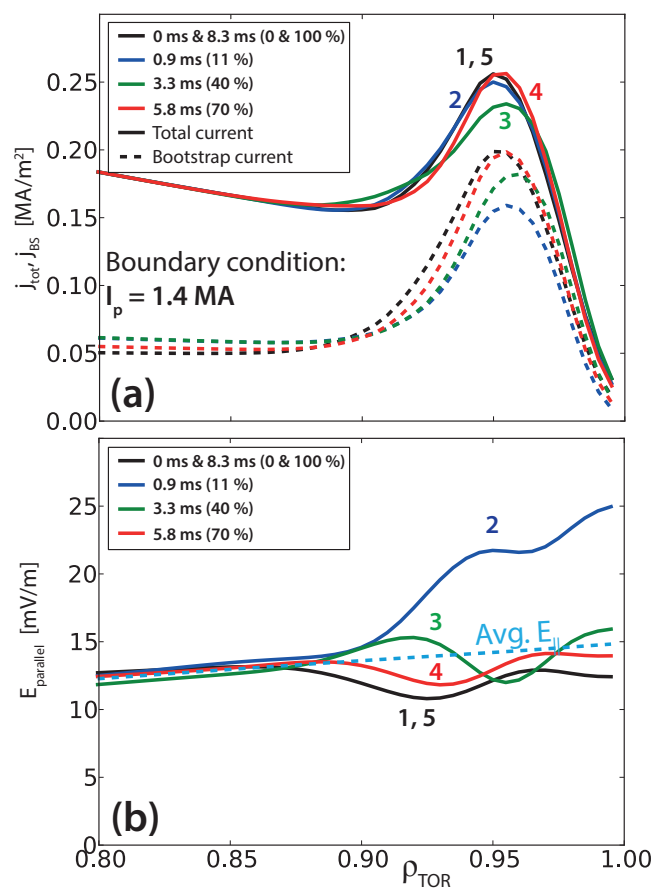

Figure 12. (a) Inter-ELM evolution of the total (solid curves) and bootstrap current (dashed curves) profiles in \#87342 with $I_{p}=$ const. boundary condition. (b) InterELM evolution of the parallel electric field. 


\subsection{Edge current evolution at high $I_{p}$}

It is important to investigate whether the conclusions reached for the JET-ILW power and gas scans at low plasma current are still relevant for H-mode scenarios at high $I_{p}$, which are more relevant for optimising fusion performance. Thus, a representative JETILW pulse (\#92432) was chosen to examine the inter-ELM edge current evolution in pedestals at high $I_{p}$. In this pulse, good performance $\left(H_{98} \simeq 1, \beta_{N} \simeq 2.1\right)$ type I ELMy $\mathrm{H}$-mode operation has been achieved at $I_{p}=3.0 \mathrm{MA}$ and $B_{t}=2.8 \mathrm{~T}$ with $\approx 33 \mathrm{MW}$ auxiliary heating.

The pedestal temperature of this discharge $\left(T_{\mathrm{e}, \mathrm{PED}}=1.1 \mathrm{keV}\right)$ and the ELM frequency $\left(f_{\mathrm{ELM}}=25 \mathrm{~Hz}\right)$ are similar to those of the highest $T_{\mathrm{e}, \mathrm{PED}}$ pulse in the low current scan (\#84794), but the pedestal top density is roughly a factor of 2 higher in \#92432 $\left(n_{\mathrm{e}, \mathrm{PED}}=5.9 \times 10^{19} \mathrm{~m}^{-1}\right)$. The pressure gradient is significantly higher in \#92432 compared to \#84794, which leads to a higher bootstrap current at the plasma edge as shown in figure 13. The inter-ELM evolution of the kinetic profiles for this discharge in the pedestal has been presented elsewhere [2]. Considering that the ELM frequency and $T_{\mathrm{e}, \mathrm{PED}}$ in \#92432 are close to those of discharge \#84794 (leading to similar conductivity, thus comparable current diffusion time scale), similar results are expected as those obtained for \#84794. Figure 13a shows the bootstrap and total edge current evolution at fixed radial position close to the peak $\left(\rho_{\mathrm{TOR}}=0.96\right)$ in the ELM cycle with $I_{\mathrm{p}}=3.0 \mathrm{MA}$ as boundary condition (in red). Similarly to the low $I_{p}$ pedestals, in this case the timescales of the total and bootstrap current evolution are also very similar in the second half of the ELM cycle.

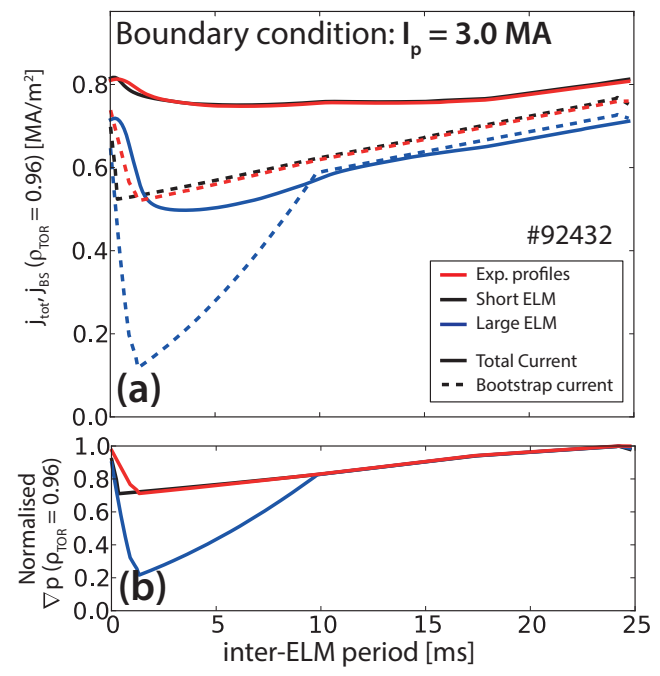

Figure 13. (a) Inter-ELM evolution of the total (solid curves) and bootstrap current (dashed curves) profiles at a given radial coordinate close to the peak $\left(\rho_{\mathrm{TOR}}=0.96\right)$ in the high $I_{p}$ discharge (\#92432) with red. Even in the case of a short ELM duration (in black) or large ELM magnitude (in blue), there is no significant delay in the total current evolution with respect to the pressure profile build-up. (b) The evolution of the peak pressure gradient in the inter-ELM cycle. 
Due to the low time resolution of the measurements, the kinetic profile evolution during the ELM crash has high uncertainties. In order to account for these uncertainties, sensitivity tests on the ELM crash duration and ELM magnitude were carried out for pulse \#92432. The nominal value of $1.5 \mathrm{~ms}$ assumed in the JETTO simulations for the ELM crash duration was determined using the ECE and interferometry measurements and it is consistent with the studies reported in [32]. In our test, the ELM duration was reduced to $400 \mu \mathrm{s}$. Figure 13 shows that changing the duration of the ELM crash from $1.5 \mathrm{~ms}$ to $400 \mu \mathrm{s}$ has no impact on the edge current evolution inter-ELM.

It is difficult to accurately quantify the ELM energy losses using the HRTS diagnostic only. Thus, the effect of an artificially increased ELM magnitude on the edge total current evolution was tested. The normalised ELM energy loss in pulse \#92432 is $\Delta W_{\mathrm{ELM}} / W_{\mathrm{PED}} \simeq 0.08$ as evaluated from the fitted kinetic profiles. In the test, this was increased with a factor of 5 to $\Delta W_{\mathrm{ELM}} / W_{\mathrm{PED}} \simeq 0.4$ in order to provoke a large drop in the bootstrap current as a result of the ELM crash. The resulting inter-ELM evolutions of $j_{\text {tot }}$ and $j_{\mathrm{BS}}$ are shown in blue in figure 13. The absolute values of the total and bootstrap currents changed significantly in the initial phase of the ELM cycle, as the average bootstrap current is reduced, however, no significant delay is found between total current and $j_{\mathrm{BS}}$ in the second half of the ELM cycle. When the ELM magnitude is large, the associated large electric field oscillation can lead to a smaller peak $j_{\text {tot }}$ than peak $j_{\text {BS }}$. This can be seen in figure 13a (blue curves) and in the case of pulse \#84794 where $\Delta W_{\mathrm{ELM}} / W_{\mathrm{PED}} \simeq 0.15$ (see figure 9 ).

\subsection{Impact of current diffusion on linear MHD pedestal stability}

Despite the small effect of current diffusion on the evolution of the total edge current density, there are some implications of the results presented in this paper on pedestal stability calculations. As a consequence of the dynamic equilibrium of bootstrap current and electric field profiles during the ELM cycle, the electric field in the pedestal is typically higher after the ELM crash and lower in the second half of the ELM cycle, compared to the fully diffused state. This could be important for pedestal linear MHD stability calculations, where usually a fully diffused electric field profile is assumed to evaluate the Ohmic contribution to the total current. Figure 14a compares the Ohmic and total edge currents for \#84794 in three different cases: the two JETTO simulations with different boundary conditions reported above and a calculation assuming fully diffused electric field profile. It can be seen that if a fully diffused electric profile is assumed, the peak total edge current is overestimated by $\sim 15-20 \%$ if $I_{p}$ is held constant and by $\sim 5-10 \%$ if the loop voltage is held constant. Although this uncertainty is small compared to other uncertainties associated with edge stability analysis, this exercise shows that the boundary condition affects the absolute value of the electric field profile and the systematic error introduced in calculations assuming a fully diffused electric field profile cannot be precisely quantified without the knowledge of the experimental boundary condition. 

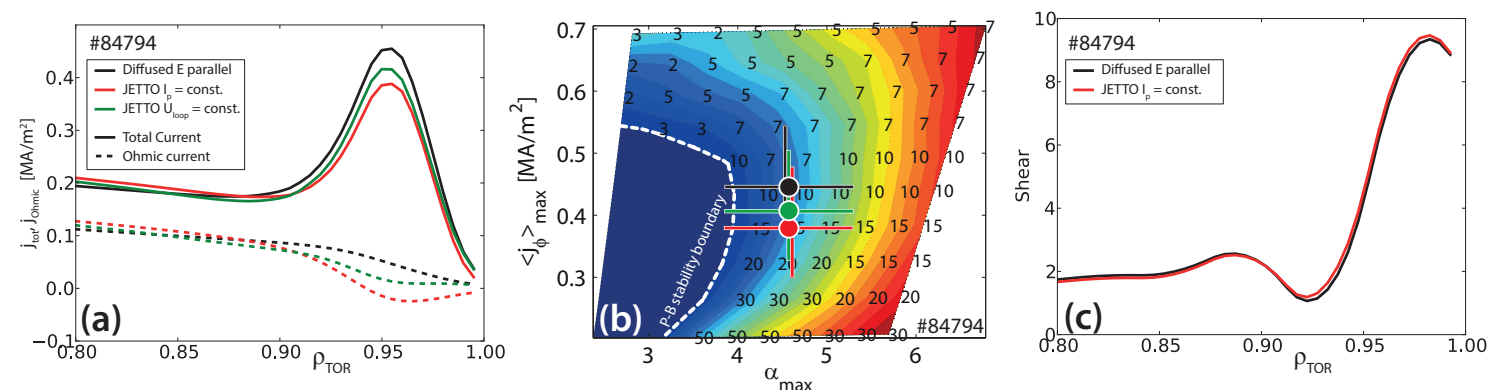

Figure 14. (a) Comparison of the edge total and Ohmic currents in the last $20 \%$ of the ELM cycle in the highest $T_{\mathrm{e}, \mathrm{PED}}$ pulse $(\# 84794): j_{\text {tot }}$ (solid lines) and $j_{\mathrm{OH}}$ (dashed lines) when the Ohmic contribution is calculated assuming a fully diffused electric field profile (black); $j_{\text {tot }}$ and $j_{\mathrm{OH}}$ when simulated in JETTO using constant total current (red) and constant loop voltage (green) as boundary condition, respectively. (b) Result of linear MHD stability analysis for \#84794. The operational point was scaled to show the impact of current diffusion on P-B mode stability in the pre-ELM phase. The y axis in figure $14 \mathrm{~b}$ is the maximum of the toroidal component of the total current density, the $\mathrm{x}$ axis is the ballooning $\alpha$. (c) The magnetic shear from the JETTO simulation with fixed $I_{p}$ (red) and from the fully diffused electric field assumption (black) are compared.

Figure 14b shows a linear MHD stability diagram calculated using the HELENA/MISHKA [33] codes for the pre-ELM phase of \#84794. The black circle shows the operational point as calculated with the HELENA equilibrium code using the input kinetic profiles, the Sauter-formula for the bootstrap current\| and assuming fully diffused electric field (this corresponds to the black curves in figure 14a). The red and green circles show the operational point for the JETTO fixed $I_{p}$ and fixed $U_{\text {loop }}^{\text {sep. }}$ simulations, respectively. The edge stability analysis was not self-consistently recalculated using the current profile outputted from JETTO, but the operational point was scaled according to the peak of the current profiles in figure 14a. As the shape of the total current density profile does not change significantly between the different cases in figure 14a, this is a good approximation to show the impact of current diffusion on $\mathrm{P}-\mathrm{B}$ mode stability in the pre-ELM phase. As it can be seen in figure 14b, the impact of current diffusion is small compared to the uncertainties arising from the profile measurement indicated by the error bars. The error bars on the operational points were calculated with a sensitivity analysis as explained in section 2 . The effect of current diffusion on the magnetic shear is negligible $(\simeq 5 \%$ at the peak of the total edge current density, $\left.\rho_{\mathrm{TOR}}=0.95\right)$. Figure $14 \mathrm{c}$ shows the shear at the plasma edge from the JETTO simulation with fixed $I_{p}$ in red and from the fully diffused electric field assumption in black. This comparison is outputted from the simulation shown in

\| The equilibrium for the ELITE stability analysis is calculated with the HELENA code in a selfconsistent way, namely that the bootstrap current and the equilibrium are calculated iteratively until the solution converges. The result of the Sauter formula for the calculation of $j_{\mathrm{BS}}$ is in good agreement with that of NEO for the low collisionality pedestal of \#84794. Thus, the usage of the Sauter formula in HELENA provides a sufficiently accurate and quick way to produce the equilibrium in this case. 
figure 10: the red curve corresponds to the end of ELM cycle, the black curve shows the magnetic shear $100 \mathrm{~ms}$ later when the electric field is fully diffused at the edge.

\section{Conclusions}

The inter-ELM evolution of the edge current density has been studied in JET-ILW type I ELMy H-mode pedestals of varying collisionality and total plasma current. The bootstrap current density, which contributes most to the total edge current, has been evaluated with the neoclassical transport code NEO. In JET-ILW type I ELMy H-modes at low gas rate the peak $j_{B S}$ is found to continuously increase during the ELM cycle. In contrast, with increasing gas rate the peak $j_{B S}$ tends to saturate during the ELM cycle, in agreement with previous analysis in JET-C [34]. The time evolution of $j_{B S}$ closely follows that of $\nabla p_{e}$, as expected, and $\nabla p_{e}$ is dominated by the $n_{e} \nabla T_{e}$ term.

The effect of current diffusion on the total edge current density has been investigated with the JETTO transport code. JET-ILW pedestals with varying $T_{\mathrm{e}, \mathrm{PED}}$ and ELM frequencies have been investigated in detail. The simulations show that there is no significant delay of the total edge current evolution with respect to the build-up of the pressure gradient inter-ELM. Sensitivity tests indicate that the conclusions are robust against ELM crash duration and/or ELM amplitude.

Although the current diffusion simulations show that the resistive timescale is comparable to the ELM period in the investigated pedestals, when the full ELM cycle (including the ELM crash) is simulated, the Ohmic current is always redistributed so as to mitigate the effect of the varying bootstrap current. As a result, the effect of current diffusion on the time evolution of the total edge current is not significant in the second half of the ELM cycle. Therefore, inter-ELM current diffusion does not explain why JET-ILW pedestals at high gas rate and high $\beta_{N}$ are stable to P-B modes, as found by linear MHD stability analysis with HELENA/ELITE [1].

One caveat of the simulations with JETTO is that the plasma shape and size are fixed during the simulation. Although the effect of total plasma current loss was investigated in the simulations with constant $U_{\text {loop }}^{\text {sep. }}$, no current loss through filaments or fast loss of a current-carrying plasma layer during the ELM crash were modelled.

Accurate modelling of the total edge current profile evolution requires a precise knowledge of the evolution of the kinetic profiles (including the ELM crash) and an accurate measurement of the loop voltage at the separatrix for the boundary condition. Without these it is difficult to quantify the effect of the Ohmic current contribution on pedestal stability analysis, although some qualitative conclusions can be made. Generally, linear MHD pedestal stability calculations estimate the contribution of the Ohmic current by assuming a fully diffused electric field profile. However, as a result of the dynamic equilibrium of the bootstrap current and electric field profiles in the ELM cycle as shown in this paper, the electric field in the pedestal is typically larger in the early phase, and lower in the second half of the ELM cycle compared to the fully diffused state. Therefore, the assumption of a fully diffused electric field may overestimate the 
total current in the pre-ELM phase. Our work estimates this error to be of order of 10$20 \%$ at the maximum of the total current density profile in the pedestal. Although, this uncertainty error is not large, it adds to all other uncertainties that feed into the edge stability analysis, such as those arising from the profile measurements and the bootstrap current models. In addition, as a consequence of the large profile changes in the first half of the ELM cycle, the fully diffused electric field assumption can potentially lead to higher errors in this phase. This might have an impact on pedestal stability analysis such as presented in $[34,35]$, where the stability of the $n=\infty$ ideal MHD ballooning mode as a proxy for Kinetic Ballooning Mode was investigated during the inter-ELM cycle.

\section{Acknowledgments}

The authors would like to thank J. Hobirk, I. Lupelli, T. Osborne, E. Solano and G. Szepesi for fruitful discussions. The authors are grateful to E. Belli and J. Candy for providing access to the NEO code. This work was supported by the Engineering and Physical Sciences Research Council [EP/L01663X/1]. This work has been carried out within the framework of the EUROfusion Consortium and has received funding from the Euratom research and training programme 2014-2018 under grant agreement No 633053. The views and opinions expressed herein do not necessarily reflect those of the European Commission.

\section{References}

[1] C.F. Maggi, S. Saarelma, F.J. Casson, C. Challis, E. de la Luna, L. Frassinetti, C. Giroud, E. Joffrin, J. Simpson, M. Beurskens, I. Chapman, J. Hobirk, M. Leyland, P. Lomas, C. Lowry, I. Nunes, F. Rimini, A.C.C. Sips, and H. Urano. Pedestal confinement and stability in JET-ILW ELMy H-modes. Nuclear Fusion, 55(11), 2015.

[2] C.F. Maggi, L. Frassinetti, L. Horvath, A. Lunniss, S. Saarelma, H. Wilson, J. Flanagan, M. Leyland, I. Lupelli, S. Pamela, H. Urano, L. Garzotti, E. Lerche, I. Nunes, F. Rimini, and JET Contributors. Studies of the pedestal structure and inter-ELM pedestal evolution in JET with the ITER-like wall. Nuclear Fusion, 57(11):116012, 2017.

[3] P. B. Snyder, H. R. Wilson, J. R. Ferron, L. L. Lao, A. W. Leonard, T. H. Osborne, A. D. Turnbull, D. Mossessian, M. Murakami, and X. Q. Xu. Edge localized modes and the pedestal: A model based on coupled peeling-ballooning modes. Physics of Plasmas, 9(5):2037-2043, 2002.

[4] H. R. Wilson, P. B. Snyder, G. T. A. Huysmans, and R. L. Miller. Numerical studies of edge localized instabilities in tokamaks. Physics of Plasmas, 9(4):1277-1286, 2002.

[5] F. Wagner, G. Becker, K. Behringer, D. Campbell, A. Eberhagen, W. Engelhardt, G. Fussmann, O. Gehre, J. Gernhardt, G. v. Gierke, G. Haas, M. Huang, F. Karger, M. Keilhacker, O. Klüber, M. Kornherr, K. Lackner, G. Lisitano, G. G. Lister, H. M. Mayer, D. Meisel, E. R. Müller, H. Murmann, H. Niedermeyer, W. Poschenrieder, H. Rapp, H. Röhr, F. Schneider, G. Siller, E. Speth, A. Stäbler, K. H. Steuer, G. Venus, O. Vollmer, and Z. Yü. Regime of improved confinement and high beta in neutral-beam-heated divertor discharges of the ASDEX tokamak. Phys. Rev. Lett., 49:1408-1412, Nov 1982.

[6] C.F. Maggi, R.J. Groebner, N. Oyama, R. Sartori, L.D. Horton, A.C.C. Sips, W. Suttrop, the ASDEX Upgrade Team, A. Leonard, T.C. Luce, M.R. Wade, the DIII-D Team, Y. Kamada, 
H. Urano, the JT-60U Team, Y. Andrew, C. Giroud, E. Joffrin, E. de la Luna, EFDAJET Contributors for the Pedestal, Edge Physics, and the Steady State Operation Topical Groups of the ITPA. Characteristics of the H-mode pedestal in improved confinement scenarios in ASDEX Upgrade, DIII-D, JET and JT-60U. Nuclear Fusion, 47(7):535, 2007.

[7] H Zohm. Edge localized modes (ELMs). Plasma Physics and Controlled Fusion, 38(2):105, 1996.

[8] K. Ikeda. Progress in the ITER physics basis. Nuclear Fusion, 47(6), 2007.

[9] A G Peeters. The bootstrap current and its consequences. Plasma Physics and Controlled Fusion, 42(12B):B231, 2000.

[10] P. B. Snyder et al. Development and validation of a predictive model for the pedestal height. Physics of Plasmas, 16(5):056118, 2009.

[11] P. B. Snyder et al. A first-principles predictive model of the pedestal height and width: development, testing and ITER optimization with the EPED model. Nuclear Fusion, 51(10):103016, 2011.

[12] M. N. A. Beurskens, J. Schweinzer, C. Angioni, A. Burckhart, C. D. Challis, I. Chapman, R. Fischer, J. Flanagan, L. Frassinetti, C. Giroud, J. Hobirk, E. Joffrin, A. Kallenbach, M. Kempenaars, M. Leyland, P. Lomas, G. Maddison, M. Maslov, R. McDermott, R. Neu, I. Nunes, T. Osborne, F. Ryter, S. Saarelma, P. A. Schneider, P. Snyder, G. Tardini, E. Viezzer, E. Wolfrum, the ASDEX Upgrade Team, and JET-EFDA Contributors. The effect of a metal wall on confinement in JET and ASDEX Upgrade. Plasma Physics and Controlled Fusion, $55(12), 2013$.

[13] A. Burckhart, E. Wolfrum, R. Fischer, K. Lackner, H. Zohm, and the ASDEX Upgrade Team. Inter-ELM behaviour of the electron density and temperature pedestal in ASDEX Upgrade. Plasma Physics and Controlled Fusion, 52(10):105010, 2010.

[14] F.M. Laggner, E. Wolfrum, M. Cavedon, M.G. Dunne, G. Birkenmeier, R. Fischer, M. Willensdorfer, F. Aumayr, The EUROfusion MST1 Team, and The ASDEX Upgrade Team. Plasma shaping and its impact on the pedestal of ASDEX Upgrade: edge stability and inter-ELM dynamics at varied triangularity. Nuclear Fusion, 58(4):046008, 2018.

[15] A. Diallo, R. J. Groebner, T. L. Rhodes, D. J. Battaglia, D. R. Smith, T. H. Osborne, J. M. Canik, W. Guttenfelder, and P. B. Snyder. Correlations between quasi-coherent fluctuations and the pedestal evolution during the inter-edge localized modes phase on DIII-D. Physics of Plasmas, $22(5): 056111,2015$.

[16] A. Diallo, J.W. Hughes, S-G. Baek, B. LaBombard, J. Terry, I. Cziegler, A. Hubbard, E. Davis, J. Walk, L. Delgado-Aparicio, M.L. Reinke, C. Theiler, R.M. Churchill, E.M. Edlund, J. Canik, P. Snyder, M. Greenwald, A. White, and the Alcator C-Mod Team. Quasi-coherent fluctuations limiting the pedestal growth on Alcator C-Mod: experiment and modelling. Nuclear Fusion, 55(5):053003, 2015.

[17] M.G. Dunne, P.J. McCarthy, E. Wolfrum, R. Fischer, L. Giannone, A. Burckhart, and the ASDEX Upgrade Team. Measurement of neoclassically predicted edge current density at ASDEX Upgrade. Nuclear Fusion, 52(12):123014, 2012.

[18] E. A. Belli and J. Candy. Kinetic calculation of neoclassical transport including self-consistent electron and impurity dynamics. Plasma Physics and Controlled Fusion, 50(9), 2008.

[19] E. A. Belli and J. Candy. Full linearized Fokker-Planck collisions in neoclassical transport simulations. Plasma Physics and Controlled Fusion, 54(1), 2012.

[20] G. Cenacchi and A. Taroni. JETTO: A free-boundary plasma transport code. Rep. JET Joint Undertaking, Abingdon, JET-IR(88)03, 1988.

[21] O. Sauter, C. Angioni, and Y. R. Lin-Liu. Neoclassical conductivity and bootstrap current formulas for general axisymmetric equilibria and arbitrary collisionality regime. Physics of Plasmas, 6(7):2834-2839, 1999.

[22] O. Sauter, C. Angioni, and Y. R. Lin-Liu. Erratum: Neoclassical conductivity and bootstrap current formulas for general axisymmetric equilibria and arbitrary collisionality regime [Phys. Plasmas 6, 2834 (1999)]. Physics of Plasmas, 9(12):5140-5140, 2002. 
[23] L. Frassinetti, M. N. A. Beurskens, R. Scannell, T. H. Osborne, J. Flanagan, M. Kempenaars, M. Maslov, R. Pasqualotto, M. Walsh, and JET-EFDA Contributors. Spatial resolution of the JET Thomson scattering system. Review of Scientific Instruments, 83(1), 2012.

[24] M. J. Leyland, M. N. A. Beurskens, J. C. Flanagan, L. Frassinetti, K. J. Gibson, M. Kempenaars, M. Maslov, R. Scannell, and JET Contributors. Edge profile analysis of Joint European Torus (JET) Thomson scattering data: Quantifying the systematic error due to edge localised mode synchronisation. Review of Scientific Instruments, 87(1), 2016.

[25] R. Scannell, M. Beurskens, P. G. Carolan, A. Kirk, M. Walsh, T. O'Gorman, and T. H. Osborne. Deconvolution of Thomson scattering temperature profiles. Review of Scientific Instruments, $82(5), 2011$.

[26] E. Stefanikova, L. Frassinetti, S. Saarelma, A. Loarte, I. Nunes, P. Lomas, F. Rimini, P. Drewelow, L. Garzotti, U. Kruezi, B. Lomonowski, E. de la Luna, L. Meneses, M. Peterka, B. Viola, and JET contributors. Effect of the relative shift between the electron density and temperature pedestal position on the pedestal stability in JET-ILW. In 43rd EPS Conference on Plasma Physics, Leuven, page O4.117, 2016.

[27] E. Stefanikova, L. Frassinetti, S. Saarelma, A. Loarte, I. Nunes, L. Garzotti, P. Lomas, F. Rimini, P. Drewelow, U. Kruezi, B. Lomanowski, E. de la Luna, L. Meneses, M. Peterka, B. Viola, C. Giroud, C. Maggi, and JET contributors. Effect of the relative shift between the electron density and temperature pedestal position on the pedestal stability in JET-ILW and comparison with JET-C. Nuclear Fusion, 58(5):056010, 2018.

[28] R. Hager and C. S. Chang. Gyrokinetic neoclassical study of the bootstrap current in the tokamak edge pedestal with fully non-linear Coulomb collisions. Physics of Plasmas, 23(4):042503, 2016.

[29] M. Romanelli, G. Corrigan, V. Parail, S. Wiesen, R. Ambrosino, P. Da Silva, L. Garzotti, D. Harting, F. Kochl, T. Koskela, L. Lauro-Taroni, C. Marchetto, M. Mattei, E. MilitelloAsp, M. F. F. Nave, S. Pamela, A. Salmi, P. Strand, G. Szepesi, and EFDA-JET Contributors. JINTRAC: A System of Codes for Integrated Simulation of Tokamak Scenarios. Plasma and Fusion Research, 9:3403023-3403023, 2014.

[30] W. A. Houlberg, K. C. Shaing, S. P. Hirshman, and M. C. Zarnstorff. Bootstrap current and neoclassical transport in tokamaks of arbitrary collisionality and aspect ratio. Physics of Plasmas, 4(9):3230-3242, 1997.

[31] G. V. Pereverzev and P. N. Yushmanov. ASTRA: Automated System for TRansport Analysis. IPP Report, Max Planck Institute for Plasmaphysics, Garching, IPP 5/98, 2002.

[32] L. Frassinetti, D. Dodt, M.N.A. Beurskens, A. Sirinelli, J.E. Boom, T. Eich, J. Flanagan, C. Giroud, M.S. Jachmich, M. Kempenaars, P. Lomas, G. Maddison, C. Maggi, R. Neu, I. Nunes, C. Perez von Thun, B. Sieglin, M. Stamp, and JET-EFDA Contributors. Effect of nitrogen seeding on the energy losses and on the time scales of the electron temperature and density collapse of type-I ELMs in JET with the ITER-like wall. Nuclear Fusion, 55(2):023007, 2015.

[33] A. B. Mikhailovskii, G. T. A. Huysmans, W. O. K. Kerner, and S. E. Sharapov. Optimization of computational MHD normal-mode analysis for tokamaks. Plasma Physics Reports, 23:844-857, 1997.

[34] S. Saarelma, M.N.A. Beurskens, D. Dickinson, L. Frassinetti, M.J. Leyland, C.M. Roach, and EFDA-JET Contributors. MHD and gyro-kinetic stability of JET pedestals. Nuclear Fusion, $53(12), 2013$.

[35] C. Bowman, D. Dickinson, L. Horvath, A.E. Lunniss, H.R. Wilson, I. Cziegler, L. Frassinetti, K. Gibson, A. Kirk, B. Lipschultz, C.F. Maggi, C.M. Roach, S. Saarelma, P.B. Snyder, A. Thornton, A. Wynn, and JET Contributors. Pedestal evolution physics in low triangularity JET tokamak discharges with ITER-like wall. Nuclear Fusion, 58(1):016021, 2018. 